\title{
Correction: A human bone marrow mesodermal-derived cell population with hemogenic potential
}

\author{
Saloomeh Mokhtari ${ }^{1} \cdot$ Evan Colletti ${ }^{2} \cdot$ Weihong Yin $^{1} \cdot$ Chad Sanada $^{3} \cdot$ Zanetta Lamar $^{1} \cdot$ Paul J. Simmons $^{4}$. \\ Steven Walker ${ }^{1} \cdot$ Colin Bishop $^{1} \cdot$ Anthony Atala $^{1}$ - Esmail D. Zanjani ${ }^{2} \cdot$ Christopher D. Porada $^{1}$. \\ Graça Almeida-Porada (iD ${ }^{1}$
}

Published online: 11 June 2018

(c) Macmillan Publishers Limited, part of Springer Nature 2018

Correction to: Leukemia https://doi.org/10.1038/s41375018-0016-1; published online: 2 February 2018

At the time of publication the below funding information was omitted from the article - this has now been corrected in both the HTML and the PDF. The publisher apologizes for any inconvenience caused.
Funding

This work was supported by NHLBI R01HL097623 and Intramural Pilot program of Wake Forest School of Medicine.

Graça Almeida-Porada

galmeida@wakehealth.edu

1 Wake Forest Institute for Regenerative Medicine, WinstonSalem, NC 27157, USA

2 Animal Biotechnology, University of Nevada Reno, Reno, NV 89557, USA

3 Currently at Fluidigm Corporation, San Francisco, CA, USA

4 Institute of Molecular Medicine, University of Texas at Houston, Houston, TX 77030, USA 